\title{
The anatomic characteristics of uterine and novel transplantation model: the experimental research
}

\author{
Do Xuan Hai ${ }^{1}$, Nguyen Trung Chuc ${ }^{1}$, Nguyen Thi Hoa ${ }^{2}$, Le Thi Hong Van ${ }^{3}$, Ngo Tuan Anh ${ }^{4}$ \\ ${ }^{1}$ Department of Surgery, Practical and Experimental Surgery, Military Medical University, Hanoi, Vietnam \\ ${ }^{2}$ Polyclinic and Premier Healthcare Center, 108 Military Central Hospital, Hanoi, Vietnam \\ ${ }^{3}$ Department of Obstetric Surgery, Military Medical University, Hanoi, Vietnam \\ ${ }^{4}$ Department of Cardiac and Vascular Surgery, 108 Military Central Hospital, Hanoi, Vietnam
}

Background: Infertility affects $10 \%-15 \%$ of couples of reproductive age. In this population, one in 500 women has absolute uterine infertility. This study was conducted to verify the uterine anatomy and a new living donor of uterine transplantation model on dog.

Methods: Anatomical study: The vascular structure of uterus was carefully dissected under magnification with surgical loupes. New uterine transplant model: We intended to examine the possibility of a living donor uterine transplantation. The uterine artery and vein were transected after the branch suppling to bladder. This plastic step was done with four segments of subcutaneous vein anastomosed to both side of uterine artery and vein with 9-0 interrupted suture. First, the vagina then the vascular component of the graft was reconnected with those on the recipient with 9-0 non absorbable suture in end to end fashion under 10x microscopic lens. The transplanted uterus was monitored in 3 hours. The histological examination of transplanted uterus was checked for signs of injury.

Results: The uterus consists of a short cervix and body and two long horns. There were two arteries on both side which supplied with blood for the uterine body and horn. The uterine artery was cranial branch of the vaginal artery. The diameter of uterine vascular pedicle was small in diameter (1-1.5 mm for arteries and 1.2-2.0 for veins) which need to be manipulated under microscope. The ovarian arteries because of extremely small size which was only $0.3-0.5 \mathrm{~mm}$. Animals living after transplantation with the rate of $100 \%$. Macro morphology of uterus after reperfusion, micro-assessment (under microscope) and histology of transplanted uterus: Uterus after transplantation has a good survival rate of $86.7 \%$. Only one case of vascular occlusion and one case of low blood pressure.

Conclusions: The living donor uterine transplantation model was feasible with the survival of transplanted uterus recognized in most cases (13/15). 